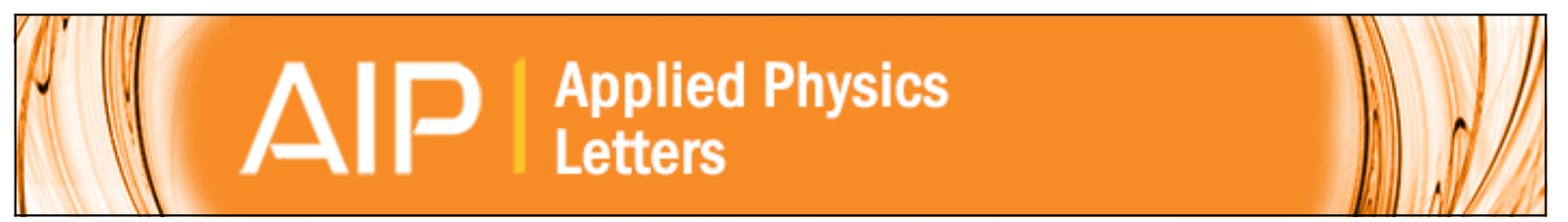

\title{
Control of waveguide properties by tuning femtosecond laser induced compositional changes
}

Jesús del Hoyo, Rebeca Martinez Vazquez, Belén Sotillo, Toney Teddy Fernandez, Jan Siegel, Paloma

Fernández, Roberto Osellame, and Javier Solis

Citation: Applied Physics Letters 105, 131101 (2014); doi: 10.1063/1.4896846

View online: http://dx.doi.org/10.1063/1.4896846

View Table of Contents: http://scitation.aip.org/content/aip/journal/apl/105/13?ver=pdfcov

Published by the AIP Publishing

\section{Articles you may be interested in}

Optical characterization of femtosecond laser induced active channel waveguides in lithium fluoride crystals

J. Appl. Phys. 115, 023108 (2014); 10.1063/1.4861636

Fabrication and characterization of periodically poled lithium niobate waveguide using femtosecond laser pulses Appl. Phys. Lett. 92, 231106 (2008); 10.1063/1.2945275

Fabrication of waveguides in Foturan by femtosecond laser

J. Laser Appl. 18, 320 (2006); 10.2351/1.2355525

Femtosecond laser-induced refractive index modification in multicomponent glasses

J. Appl. Phys. 97, 083102 (2005); 10.1063/1.1876578

Waveguide fabrication in phosphate glasses using femtosecond laser pulses

Appl. Phys. Lett. 82, 2371 (2003); 10.1063/1.1565708 \section{AIP | Applied Physics \\ is pleased to announce Reuben Collins as its new Editor-in-Chief}




\title{
Control of waveguide properties by tuning femtosecond laser induced compositional changes
}

\author{
Jesús del Hoyo, ${ }^{1}$ Rebeca Martinez Vazquez, ${ }^{2}$ Belén Sotillo, ${ }^{3}$ Toney Teddy Fernandez, ${ }^{1}$ \\ Jan Siegel, ${ }^{1}$ Paloma Fernández, ${ }^{3}$ Roberto Osellame, ${ }^{2}$ and Javier Solis ${ }^{1, a)}$ \\ ${ }^{1}$ Laser Processing Group, Instituto de Optica, CSIC, Serrano 121, 28006 Madrid, Spain \\ ${ }^{2}$ Instituto di Fotonica e Nanotecnologie, CNR, Piazza Leonardo da Vinci 32, 20133 Milano, Italy \\ ${ }^{3}$ Depto. de Física de Materiales, Facultad de Físicas, Univ. Complutense, 28040 Madrid, Spain
}

(Received 1 August 2014; accepted 15 September 2014; published online 29 September 2014)

\begin{abstract}
Local compositional changes induced by high repetition rate fs-laser irradiation can be used to produce high performance optical waveguides in phosphate-based glasses. The waveguide refractive index contrast is determined by the local concentration of $\mathrm{La}$, which can be changed by the action of the writing laser pulses. In this work, we have investigated the degree of control that can be exerted using this waveguide writing mechanism over the cross-section of the guiding region, and the local refractive index and compositional changes induced. These variables can be smoothly controlled via processing parameters using the slit shaping technique with moderate Numerical Aperture (NA 0.68) writing optics. The combined use of X-ray microanalysis and near field refractive index profilometry evidences a neat linear correlation between local La content and refractive index increase over a broad $\Delta \mathrm{n}$ interval $\left(>3 \times 10^{-2}\right)$. This result further confirms the feasibility of generating efficient, integrated optics elements via spatially selective modification of the glass composition. @ 2014 AIP Publishing LLC. [http://dx.doi.org/10.1063/1.4896846]
\end{abstract}

Femtosecond (fs) laser writing ${ }^{1}$ has been proven a versatile method for producing optical waveguides inside both glassy $^{2}$ and crystalline dielectrics, ${ }^{3}$ including highly nonlinear ones. ${ }^{4}$ Yet, one of its present limitations is imposed by the maximum refractive index contrast accessible, which is given by the refractive index modification mechanism involved. Refractive index changes upon fs-laser writing have been ascribed to a variety of mechanisms including lattice densification/expansion, ${ }^{5,6}$ point defects, ${ }^{7}$ photochemical modifications of the glass network, ${ }^{8}$ and changes in the polarizability of the glass. ${ }^{9}$ However, with just a few exceptions, ${ }^{10,11}$ the associated refractive index change $(\Delta \mathrm{n})$ is below $10^{-2}$ and commonly involves the use of oilimmersion, high Numerical Aperture (NA), writing optics. The feasibility of inducing controlled migration of ions by fs-laser irradiation would thus open up additional prospects for fabricating efficient integrated optical devices inside glasses as envisaged in Ref. 12.

Indeed, the observation of local compositional changes upon irradiation of glasses with high and low repetition rate fs-laser pulses has been a subject of study since the early work of Hirao and co-workers regarding the precipitation of non-linear crystals in glass matrices. ${ }^{13}$ More recently, element redistribution has been observed upon static ${ }^{14,15}$ or dynamic $^{16,17}$ (moving sample) irradiation experiments mostly in silicate glasses. However, none of these works has provided evidence of local compositional changes leading to light guiding in the written structures. Only recently, it has been demonstrated that local modifications of the glass composition can be used to produce extremely efficient active/ passive waveguides in homemade ${ }^{18}$ and commercial phosphate glasses ${ }^{19}$ with $\mathrm{Al}_{2} \mathrm{O}_{3}, \mathrm{~K}_{2} \mathrm{O}$, and $\mathrm{La}_{2} \mathrm{O}_{3}$ glass modifiers.

\footnotetext{
${ }^{\text {a) }}$ Author to whom correspondence should be addressed. Electronic mail: j.solis@io.cfmac.csic.es
}

The refractive index changes were due to the cross migration of $\mathrm{La}$ and $\mathrm{K}$ ions, leading to $\Delta \mathrm{n} \sim 1.5 \times 10^{-2}$ at $1620 \mathrm{~nm}$. Although smaller in magnitude $\left(\Delta \mathrm{n} \sim 3.5 \times 10^{-3}\right.$ at $\left.670 \mathrm{~nm}\right)$, cross migration of $\mathrm{Te}$ and $\mathrm{Na}$ ions has been shown to assist local refractive index modifications also in a tellurite glass. ${ }^{20}$

In this work, we analyze the degree of control that can be exerted over the cross section, refractive index, and compositional features of waveguides written via fs-laser induced ion migration in a phosphate glass, in which La acts as the refractive index control element.

The phosphate glass sample used is made of KigreQxErSpa100 (composition $\sim 65 \cdot \mathrm{P}_{2} \mathrm{O}_{5}-10 \cdot \mathrm{La}_{2} \mathrm{O}_{3}-10 \cdot \mathrm{Al}_{2} \mathrm{O}_{3}-$ 10. $\mathrm{K}_{2} \mathrm{O}$ mol. \%) doped with $\mathrm{Er}_{2} \mathrm{O}_{3}\left(2\right.$ wt. \%) and $\mathrm{Yb}_{2} \mathrm{O}_{3}$ ( 4 wt. \%). The waveguides were written using a slit-shaped laser beam generated by high repetition rate, femtosecond laser amplifier (Tangerine, Amplitude Systems) operating at a wavelength of $1030 \mathrm{~nm}$ and a repetition rate of $500 \mathrm{kHz}$. The pulse duration was $\sim 400 \mathrm{fs}$ and the beam diameter before the slit was $4.3 \mathrm{~mm}\left(1 / \mathrm{e}^{2}\right.$ diameter). The structures were produced $100 \mu \mathrm{m}$ underneath the surface using a $0.68 \mathrm{NA}$ aspheric lens. As variable processing parameters for the study, we used the sample scan speed $(v=40,60$, and $80 \mu \mathrm{m} / \mathrm{s}$ ), slit width (no slit, $s=1.2$ and $1.4 \mathrm{~mm}$ ), and the pulse energy reaching the surface $(500-800 \mathrm{~nJ} / \mathrm{pulse})$. Further details on the writing set-ups are given elsewhere. ${ }^{18,19}$

Figure 1 shows several transmission optical microscopy images (differential interference contrast mode, DIC) of waveguides produced with and without slit at different pulse energies measured after the slit. In all cases, the waveguide presents a white nearly circular region on top of a dark pointed zone with depressed index. These two regions have been identified as caused by the cross migration of $\mathrm{La}$ and $\mathrm{K}$, leading to a modification of the refractive index, which increases with the local La content. ${ }^{19,21}$ In terms of the 


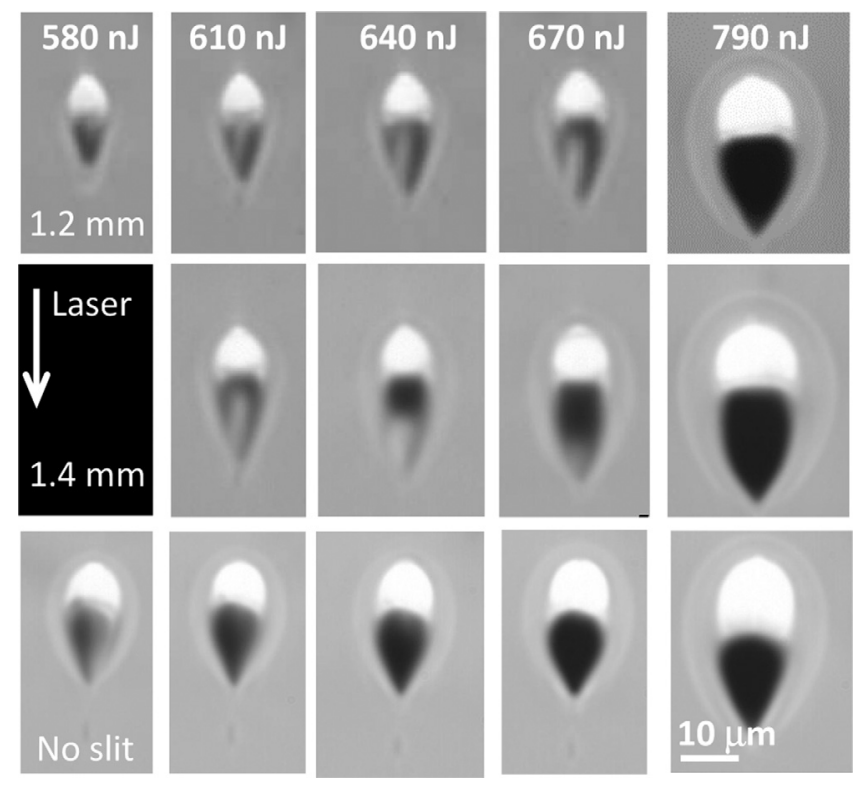

FIG. 1. Optical microscopy images of waveguides produced with ( $s=1.2 \mathrm{~mm}$ (upper row), $s=1.4 \mathrm{~mm}$ (mid row)), and without slit (lower row), at $60 \mu \mathrm{m} / \mathrm{s}$, with the indicated pulse energy. The arrow indicates the laser beam direction.

control that can be exerted over the cross-section of the structures, the most remarkable aspect of Fig. 1 is that it shows that the size of the guiding region can be fine-tuned by adjusting either the pulse energy or the slit width. The slit modifies the way energy is coupled to the material, leading to a counterintuitive result: for the same incident energy, the structures are narrower when using a slit to shape the beam.

In this irradiation conditions, the waveguides are formed due to the balance between heat accumulation and thermal diffusion $^{22}$ effects, as can be observed by comparing the cross-section dimensions (Fig. 1) with the spot size of the aspheric lens $(\sim 1 \mu \mathrm{m})$. The use of the slit modifies the shape of the focal volume by enlarging the dimension orthogonal to the slit aperture (a factor of $\sim 3$ in our conditions), thus the same pulse energy on the glass is distributed on a larger volume. The lower energy density may not trigger the nonlinear absorption in the whole focal volume (in particular, when the pulse energy is moderate) and results in smaller glass modifications when the slit is used. On the contrary, when high pulse energies $(>730 \mathrm{~nJ}$ in our conditions) are used, the energy density is well-above threshold in the whole focal volume and the energy absorption, and thus waveguide size, is almost the same irrespectively of the slit width (see Figs. 1 and 2). It can be concluded that, differently from non-thermal regimes where the waveguide cross-section closely resemble the focal volume shape, in this thermal regime, the use of the slit does not directly determine the size of the waveguide, but does so indirectly by affecting the energy absorption process.

The evolution of the size of the guiding region $(D)$ as a function of energy is shown in Figure 2 for $s=1.2 \mathrm{~mm}$, and "no-slit." Both curves show a quasi-parallel evolution for writing energies above threshold $(\sim 520 \mathrm{~nJ})$ and below $730 \mathrm{~nJ}$, with a vertical shift of $\sim 4 \mu \mathrm{m}$. This shift has an important consequence on the guiding characteristics of the structures. The waveguides produced without slit and showing $D$ values $>\sim 8 \mu \mathrm{m}$ (shaded area, corresponding to pulse

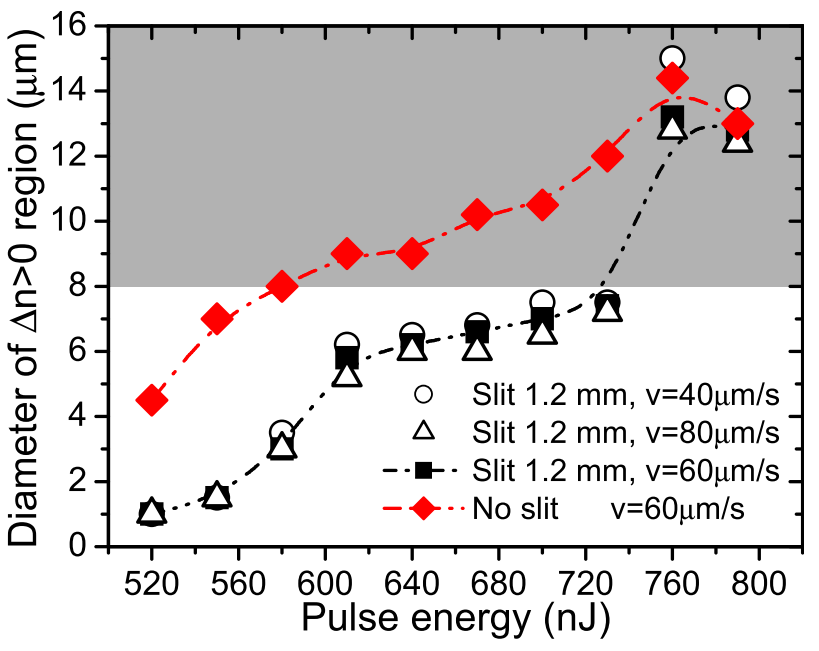

FIG. 2. Diameter of the guiding region along the direction transverse to the laser vs. pulse energy for the indicated speeds and slits. Lines are to guide the eye. The grey zone marks the region where the waveguides produced without slit are multi-mode at $980 \mathrm{~nm}$.

energy $\geq 580 \mathrm{~nJ}$ ) do not support single-mode propagation at $980 \mathrm{~nm} .{ }^{19}$ This greatly reduces the useful pulse energy interval to produce efficient active waveguides without slitshaping. Indeed, for lower pulse energies $(<580 \mathrm{~nJ})$, the produced structures are highly stressed and poorly guiding. In contrast, for $s=1.2 \mathrm{~mm}$, single mode propagation can be achieved for pulse energies up to $670 \mathrm{~nJ}$ (in this case, at $80 \mu \mathrm{m} / \mathrm{s})$. In the plot, we can also see that $D$ increases faster for energies up to $\sim 600 \mathrm{~nJ}$, while in the $610-730 \mathrm{~nJ}$ interval, the slope of the curves diminishes. The behavior for $s=1.4 \mathrm{~mm}$ (not shown) is similar to the one for $s=1.2 \mathrm{~mm}$ but slightly shifted to higher $D$ values, $\sim 0.5 \mu \mathrm{m}$ in the 610-730 nJ interval. Above $730 \mathrm{~nJ}$, the structure size strongly increases again, and $D$ finally reaches similar values for the slit-less and slit-shaped structures (see also Fig. 1).

In the studied scan speed interval, the effect of changing $v$ within a factor of 2 has only a relatively small influence over the size of the transformed region, in agreement with the observations in Ref. 22. Overall, Fig. 2 shows that the amplitude of the energy interval to fine tune the size of a structure in a given range is determined by the slit width. This effect has been shown to be particularly useful for fine tuning the dimensions of single mode waveguides both at $980 \mathrm{~nm}$ and $1530 \mathrm{~nm}$, as shown in Ref. 19.

In order to analyze the control over the compositional changes produced and the local refractive index changes achievable, the local La content of the structures was analyzed in a Leica S440 Scanning Electron Microscope (SEM) equipped with a Bruker AXS Quantax X-ray microanalysis system with a resolution of $125 \mathrm{eV}$, while the refractive index distribution in the waveguides was mapped (at $670 \mathrm{~nm}$ ) by means of a near-field refractive index profilometer (Rinck Elektronik). Figure 3 shows as an illustrative example two images of the same structure as seen by SEM and near-field refractometry. An image of the guided mode of the structure at $1620 \mathrm{~nm}$ is included for reference. The comparison with the structures in Fig. 1 clearly shows that the guiding region coincides with the white contrasted region in the optical microscopy, SEM (positive Z-contrast), and refractometry 

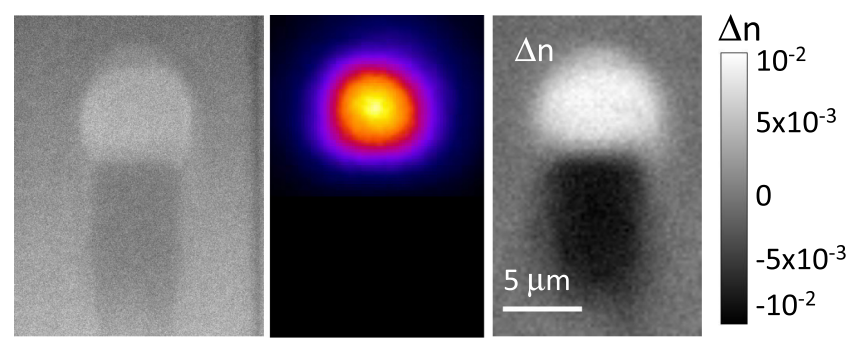

FIG. 3. Scanning electron microscope (left) and refractive index profilometry (right) images of a waveguide written at a scan speed of $60 \mu \mathrm{m} / \mathrm{s}$ with a slit width of $1.4 \mathrm{~mm}$ and $670 \mathrm{~nJ} /$ pulse. A near field image of the guided mode at $1620 \mathrm{~nm}$ (center) is included for reference.

images (positive $\Delta \mathrm{n}$ zone), consistently with the reported La enrichment in the guiding zone (La depletion in the dark zones). ${ }^{19}$ Also, the small mode field diameter observed, well below $10 \mu \mathrm{m}$ is a clear indication of the strong confinement achieved and large refractive index change induced. ${ }^{11,18,19}$

The insets of Fig. 4 show the refractive index and La-K compositional maps of the same structure. By measuring the La content by X-ray microanalysis and comparing the resulting values with the corresponding local refractive index maps, it is possible to establish a quantitative correlation between local refractive index and La-content, as shown in Fig. 4. Both parameters show a linear correlation, with $\Delta \mathrm{n}=4.95 \times 10^{-3}$ per mol fraction of La, a relation essentially similar to that estimated in Ref. 19 based on the experimental data available for binary $\mathrm{P}_{2} \mathrm{O}_{5}-\mathrm{La}_{2} \mathrm{O}_{3}$ glasses. ${ }^{21}$ This provides a very large range of accessible index contrast for the production of highly confined structures and small radius of curvature waveguides. ${ }^{23}$ The extent of this interval could potentially be broadened by modifying the initial $\mathrm{La} / \mathrm{K}$ ratio of the glass composition. An estimate of the curvature radius that could be supported by the present structures for a $1.2 \mathrm{~mm}$ slit-shaped waveguide with a positive refractive index of $1.5 \times 10^{-2}$ (Ref. 24) provides a value of $\sim 8 \mathrm{~mm}^{23}$

Although the relation between local ion concentration and refractive index is well known and modeled in the case of ion-exchanged waveguides, ${ }^{25}$ the precise correlation between local concentration and refractive index in a fs-laser

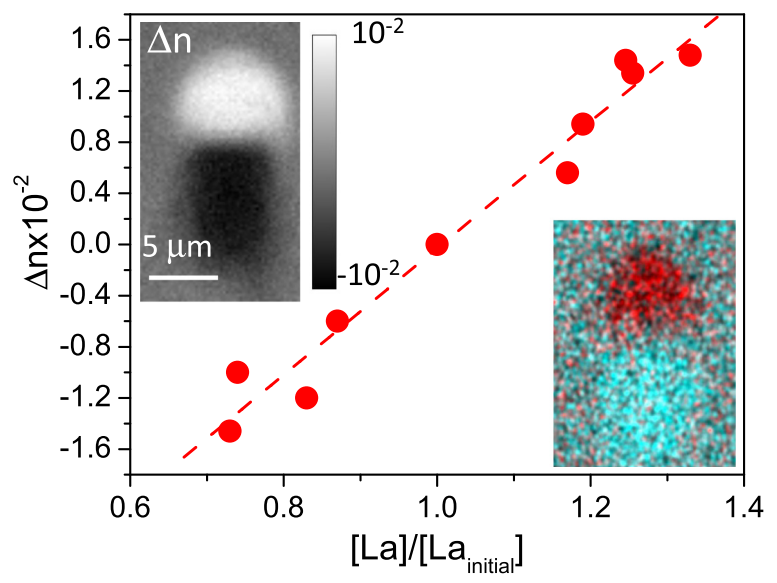

FIG. 4. Local refractive index change $(\Delta \mathrm{n})$ as a function of the variation of the La content for waveguides written with and without slit, for different pulse energies. The inset shows the refractive index and a qualitative La-K compositional mapping ( $\mathrm{La}$ in red and $\mathrm{K}$ in cyan) of the same structure shown in Fig. 3. Line is a linear fit of the data. written waveguide is a very remarkable result. It must also be noted that local variations of the $\mathrm{K}$ content could potentially contribute to the local index of the structures. However, a representation similar to that of Fig. 4 for the case of $\mathrm{K}$ leads to a strongly scattered plot in which the refractive index shows only a general decreasing trend with the $\mathrm{K}$ content (due to the correlated behavior of $\mathrm{La}$ ). In all cases, cross migration of $\mathrm{K}$ to the low index zone takes place, a well known behavior for monovalent ions, which usually diffuse towards defect rich zones. ${ }^{20}$ Nevertheless, we observed large variations of the $\mathrm{K}$ content for similar index values that further support that $\mathrm{La}$ is the refractive index control element.

Figure 5 shows the evolution of the maximum $\Delta \mathrm{n}$ values in the guiding region versus the pulse energy in structures written for different slit width values. The curve shows an overall behavior that reminds the one of the plots in Fig. 2. For energies above threshold and up to $\sim 620 \mathrm{~nJ}, \Delta \mathrm{n}$ increases moderately while the size of the structures grows rapidly. At about $620 \mathrm{~nJ}$ and up to $720 \mathrm{~nJ}$, D shows a much more moderate increase rate while $\Delta \mathrm{n}$ grows rapidly from approximately $5 \times 10^{-3}$ up to $1.4 \times 10^{-2}$. A further energy increase leads to a nearly saturated behavior, both the size of the structures and the maximum refractive index increase induced. A more detailed comparison between Figs. 2 and 5 shows that in the intermediate pulse energy region (620-720 nJ), the refractive index of the structure can be very precisely controlled via pulse energy, while its diameter can be tuned via slit width, thus providing full control over the guiding properties of the structure. It is worth stressing that maximum $\Delta \mathrm{n}$ values and single mode operation at $980 \mathrm{~nm}$ and $1530 \mathrm{~nm}$ can only be achieved simultaneously with slit shaping.

It has to be noted that the ultimate reasons for the migration of $\mathrm{La}$ towards the guiding region remain unclear. Previous works have stressed the role of thermal gradients in fs laser-induced ion migration phenomena ${ }^{14-16}$ or the opposite migration behavior of glass formers and modifiers. ${ }^{12}$ Also more recently, thermal ionization has been analyzed as an important factor in high repetition rate processing of glass. ${ }^{26}$ However, the reason for a given ion to move towards or opposite to a given gradient direction remains unsolved. Once a given ion initiates its migration in the presence of a

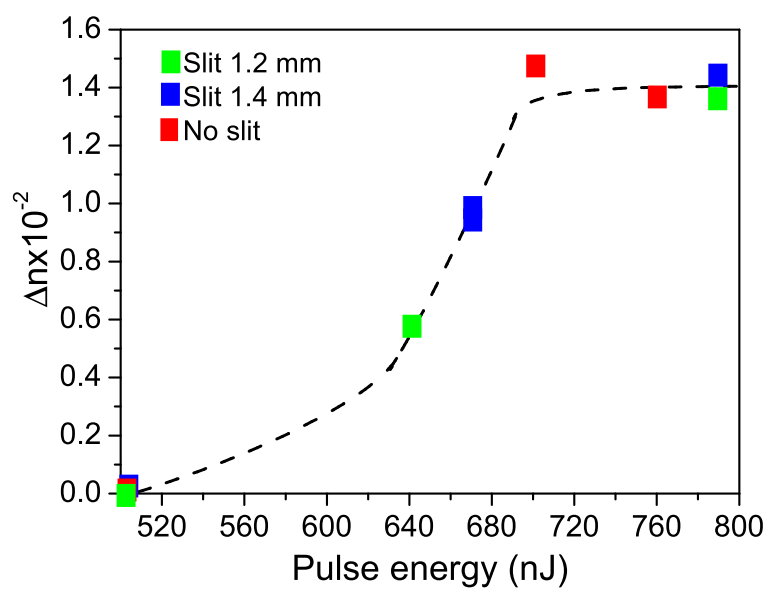

FIG. 5. Maximum local refractive index $(\Delta \mathrm{n})$ vs. pulse energy for structures produced with different slit widths. 
thermal gradient, other species should move in the opposite direction in a high-temperature, highly ionized, molten state to compensate charge/oxygen deficiencies. Further studies will be required to answer this question, hopefully providing a global model for a variety of glass materials.

In summary, we have shown that in high repetition rate fs-laser written waveguides produced by element (La, K) migration in phosphate glass, it is feasible to exert a precise control of the cross-section and refractive index of the guiding structures produced. Control is given by the use of slit shaping that modifies the energy deposition profile and the structure cross-section while pulse energy is the dominant factor conditioning the enrichment of La in the guiding zone.

This work was partially supported by the Spanish Ministry Economy and Competitiveness (MINECO, TEC2011-22422, MAT2012-31959), J.H. and T.T.F. acknowledge funding from the JAE CSIC Program (pre- and post-doctoral fellowships, respectively, co-funded by the European Social Fund). B. Sotillo acknowledges her funding in the frame of CSD2009-00013 (MINECO).

${ }^{1}$ K. M. Davis, K. Miura, N. Sugimoto, and K. Hirao, Opt. Lett. 21, 1729 (1996).

${ }^{2}$ R. Osellame, N. Chiodo, G. Della Valle, G. Cerullo, R. Ramponi, P. Laporta, A. Killi, U. Morgner, and O. Svelto, IEEE J. Sel. Top. Quantum Electron. 12, 277 (2006).

${ }^{3}$ J. Burghoff, C. Grebing, S. Nolte, and A. Tünnermann, Appl. Phys. Lett. 89, 081108 (2006).

${ }^{4}$ J. Siegel, J. Fernandez-Navarro, A. Garcia-Navarro, V. Diez-Blanco, O. Sanz, J. Solis, F. Vega, and J. Armengol, Appl. Phys. Lett. 86, 121109 (2005).

${ }^{5}$ J. W. Chan, T. Huser, S. Risbud, and D. M. Krol, "Structural changes in fused silica after exposure to focused femtosecond laser pulses," Opt. Lett. 26, 1726-1728 (2001).

${ }^{6}$ A. Ferrer, D. Jaque, J. Siegel, A. R. De la Cruz, and J. Solis, J. Appl. Phys. 109, 093107 (2011).
${ }^{7}$ K. Hirao and K. Miura, J. Non. Cryst. Solids 239, 91 (1998).

${ }^{8}$ O. Efimov, L. Glebov, K. Richardson, E. Van Stryland, T. Cardinal, S. Park, M. Couzi, and J. Bruneel, Opt. Mater. 17, 379 (2001).

${ }^{9}$ D. J. Little, M. Ams, P. Dekker, G. D. Marshall, and M. J. Withford, J. Appl. Phys. 108, 033110 (2010).

${ }^{10}$ R. Martinez-Vazquez, R. Osellame, G. Cerullo, R. Ramponi, and O. Svelto, Opt. Express 15, 12628-12635 (2007).

${ }^{11}$ S. M. Eaton, M. L. Ng, R. Osellame, and P. R. Herman, J. Non. Cryst. Solids 357, 2387 (2011).

${ }^{12}$ Y. Liu, M. Shimizu, B. Zhu, Y. Dai, B. Qian, J. Qiu, Y. Shimotsuma, K. Miura, and K. Hirao, Opt. Lett. 34, 136, (2009).

${ }^{13}$ Y. Yonesaki, K. Miura, R. Araki, K. Fujita, and K. Hirao, J. Non. Cryst. Solids 351, 885 (2005).

${ }^{14}$ M. Shimizu, M. Sakakura, S. Kanehira, M. Nishi, Y. Shimotsuma, K. Hirao, and K. Miura, Opt. Lett. 36, 2161 (2011).

${ }^{15}$ M. Sakakura, T. Kurita, M. Shimizu, K. Yoshimura, Y. Shimotsuma, N. Fukuda, K. Hirao, and K. Miura, Opt. Lett. 38, 4939 (2013).

${ }^{16}$ F. Luo, J. Song, X. Hu, H. Sun, G. Lin, H. Pan, Y. Cheng, L. Liu, J. Qiu, Q. Zhao, and Z. Xu, Opt. Lett. 36, 2125 (2011).

${ }^{17}$ P. Mardilovich, L. B. Fletcher, N. W. Troy, L. Yang, H. Huang, S. H. Risbud, and D. M. Krol, Int. J. Appl. Glas. Sci. 4, 87 (2013).

${ }^{18}$ J. Hoyo, V. Berdejo, T. T. Fernandez, A. Ferrer, A. Ruiz, J. A. Valles, M. A. Rebolledo, I. Ortega-Feliu, and J. Solis, Laser Phys. Lett. 10, 105802 (2013).

${ }^{19}$ T. T. Fernandez, P. Haro-González, B. Sotillo, M. Hernandez, D. Jaque, P. Fernandez, C. Domingo, J. Siegel, and J. Solis, Opt. Lett. 38, 5248 (2013).

${ }^{20}$ T. T. Fernandez, M. Hernandez, B. Sotillo, S. M. Eaton, G. Jose, R. Osellame, A. Jha, P. Fernandez, and J. Solis, Opt. Express 22, 15298 (2014).

${ }^{21}$ R. K. Brow, E. Metwalli, and D. L. Sidebottom, Proc. SPIE 4102, 88-94 (2000).

${ }^{22}$ S. M. Eaton, H. Zhang, M. L. Ng, J. Li, W.-J. Chen, S. Ho, and P. R. Herman, Opt. Express 16, 9443 (2008).

${ }^{23}$ A. Arriola, S. Gross, N. Jovanovic, N. Charles, P. G. Tuthill, S. M. Olaizola, A. Fuerbach, and M. J. Withford, Opt. Express 21, 2978 (2013).

${ }^{24}$ The experimental refractive index map used for the estimation had a maximum $\Delta \mathrm{n}=1.5 \times 10-{ }^{2}$ at $670 \mathrm{~nm}$, similar to the one estimated from waveguide NA measurements at $1620 \mathrm{~nm} .{ }^{19}$ The dispersion of the glass seems thus little affected by the compositional change.

${ }^{25}$ A. Tervonen, B. R. West, and H. Seppo, Opt. Eng. 50, 071107 (2011).

${ }^{26}$ M. Sun, U. Eppelt, W. Schulz, and J. Zhu, Opt. Mater. Express 3, 1716 (2013). 